\title{
A Comparison of the Pulmonary Valve, Main Pulmonary Artery, and Branch Pulmonary Artery Measurements by Echocardiography and Computed Tomography Scan
}

\author{
Priyanka A. Parikh ${ }^{1}$, Binoy V. Shah ${ }^{1}$, Bhadra Trivedi ${ }^{2}$, Viral B. Patel ${ }^{3,4,5}$, Shreya Desai ${ }^{3,4,5}$, \\ Somashekhar M. Nimbalkar 6, 1 , Ajay G. Phatak ${ }^{6}$

\begin{abstract}
1. Department of Pediatrics, Pramukhswami Medical College, Karamsad, IND 2. Pediatric Cardiology, Shree Krishna Hospital, Karamsad, IND 3. Radiodiagnosis, Pramukhswami Medical College, Karamsad, IND 4. Radiodiagnosis, Shree Bhaikaka University, Karamsad, IND
\end{abstract} \\ Krishna Hospital, Karamsad, IND 5. Radiodiagnosis, Bhaikaka University, Karamsad, IND 6. Central Research Services,
}

Corresponding author: Bhadra Trivedi, bhadra81@gmail.com

\begin{abstract}
Introduction: Congenital heart diseases (CHD) are one of the most commonly occurring congenital anomalies. Echocardiography is usually the initial investigation for suspected CHD. However, it is operatordependent and limited by available chest windows. Multidetector computed tomography (MDCT) scan provides superior temporal and spatial resolution producing excellent cross-sectional anatomical images. MDCT is specifically helpful for pulmonary artery anomalies if not clearly visible on an echocardiogram.
\end{abstract}

Objective and methods: The study aims to compare measurements of branch pulmonary arteries, pulmonary valve, and main pulmonary artery obtained from trans-thoracic echocardiography measurements and MDCT. Forty-nine patients younger than 17 years of age underwent MDCT, and an echocardiogram was included in the study. The measurements of the pulmonary valve, main pulmonary artery, and branch pulmonary arteries were measured on MDCT and echocardiogram.

Results: Bland-Altman analysis revealed the mean difference (95\% confidence limits) in measurements of diameter between echocardiogram and MDCT for the right pulmonary artery, left pulmonary artery, pulmonary valve, and main pulmonary artery, which were $-0.5(-3.1,2.2) \mathrm{mm},-0.6(-3.3,2.1) \mathrm{mm}, 0.7(-2.5$, 3.9) $\mathrm{mm}$, and $1.2(-6.9,4.5) \mathrm{mm}$, respectively.

Conclusion: The analysis revealed acceptable agreement in measurements of the pulmonary valve, main pulmonary artery, and branch pulmonary arteries obtained from MDCT and echocardiogram. The difference was marginally more for the main pulmonary artery compared to the pulmonary valve and branch pulmonary arteries.

Review began 06/01/2021 Review ended 06/24/2021 Published 06/30/2021

\section{(๑) Copyright 2021}

Parikh et al. This is an open access article distributed under the terms of the Creative Commons Attribution License CC-BY 4.0., which permits unrestricted use, distribution, and reproduction in any medium, provided the original author and source are credited.
Categories: Cardiac/Thoracic/Vascular Surgery, Cardiology, Pediatrics

Keywords: pediatrics \& neonatology, pediatric congenital heart disease, multidetector computed tomography, echocardiography - heart failure - valvular heart disease, intervention pediatric cardiology, pediatric clinical cardiology, cardiothoracic \& vascular surgery research, trans-thoracic echocardiography, computed tomography

\section{Introduction}

Congenital heart diseases (CHD) comprise 8/1000 live births and account for a high prevalence of infant mortality worldwide $[1,2]$. Trans-thoracic echocardiography (TTE) is the most preferred investigation for evaluating CHD; however, it is operator-dependent and limited by the acoustic window. Over recent years, the TTE machines have undergone a significant upgrade in terms of technological advances. Hence, the image quality of 2D TTE is reliable and reproducible in experienced hands [3]. It has the advantage of being non-invasive and radiation-free. The TTE has Doppler capabilities and can track blood cells passing through the vessels. Thus studying the anatomic and hemodynamic details without intravenous contrast is possible.

Multidetector computed tomography (MDCT) scan has been increasingly utilized for imaging the heart for various congenital and acquired cardiac anomalies. It gives superior temporal and spatial resolution producing excellent cross-sectional anatomical images. Thus, it is considered the gold standard to study static anatomic details for the cardiac structures in the pediatric population [4]. With advancements in the MDCT scan technology, axial data can generate non-axial images, such as sagittal and coronal, using multiplanar reformation (MPR). Software analysis tools like maximum intensity projection (MIP) and volume rendering (VR) techniques have improved diagnostic capabilities. Precise three-dimensional imaging obtained from the MDCT scan help in planning the treatment for congenital and acquired cardiac pathologies [5]. The drawbacks of the MDCT scan are the inevitable radiation doses and the use of a contrast medium, which is essential to assess the anatomy of smaller vessels. 
Accurate preoperative assessment of pulmonary artery anatomy influences the surgical approach and aids in treatment planning [6]. The anatomy of the pulmonary valve, main pulmonary artery, and branch pulmonary arteries are the significant components of evaluation in cases of suspected congenital heart disease as per recommended guidelines [7]. Adequate information is received from the echocardiogram most of the time. Computed tomography (CT) scan pulmonary angiogram enhances the accuracy of measurements and compliments the echocardiogram findings. The study aims to assess whether TTE measurements and MDCT measurements of branch pulmonary arteries, pulmonary valve, and main pulmonary artery are comparable.

\section{Materials And Methods}

This retrospective study was approved by the Institutional Review Board based on a medical record review. Forty-nine pediatric patients who underwent MDCT pulmonary angiogram and echocardiogram were included in the study. Each case was discussed among the authors to ensure that the patients' clinical condition had not changed between the MDCT and TTE, which would otherwise affect the sizes of great vessels. If either of the study modality MDCT or TTE were sub-optimal or missing, the cases were excluded. Information about the demographic profile, including the cardiac disease, was gathered without personal identifiers. The TTE evaluation was performed using the Siemens Acuson x300 USG machine (Siemens, Munich, Germany) using Siemens Acuson P8-4 phased array sector. It was performed by the same pediatric cardiologist every time following a standard protocol in a dedicated echocardiogram lab. Whenever a child was not cooperative and sedation was required, Trichlophos oral solution was used with $50 \mathrm{mg} / \mathrm{kg}$ single dose. Patients were observed in the nursing station in the outpatient department (OPD) area until the child was fully awake. The measurements of the pulmonary valve were done using the left parasternal long-axis view. The main pulmonary artery and branch pulmonary arteries were measured in the left parasternal short-axis view in systole as per recommendation [8]. The maximum diameter was considered for measurements. The MDCT scans were performed on GE Healthcare Optima CT 660 (GE Healthcare, Chicago, Illinois). The normal value of serum creatinine was mandatory before each MDCT scan.

A combination of injectable ketamine, midazolam, and glycopyrrolate was used for sedation during the MDCT scan as per the protocols followed by the pediatric cardiac intensivist. Patients were examined in a supine position, and all scans extended from the nape to the aortic bifurcation in a craniocaudal direction to assess abdominal vascular structures and viscera. Non-ionic iodinated contrast material (Vizipaque 270 $\mathrm{mg} / \mathrm{ml}$ ) was given intravenously by a pressure injector at a rate usually varying from $2 \mathrm{ml} / \mathrm{s}$ to $2.5 \mathrm{ml} / \mathrm{s}$. The total dose was kept at $2 \mathrm{ml} / \mathrm{kg}$ of body weight. Saline bolus injection followed contrast with the same volume and rate. Since the access route was not fixed in all patients, e.g., arm or leg, a modified bolus tracking setting was used depending on access. The scan was initiated as soon as the contrast was visible in the upper part of descending aorta. The acquisition parameters used for CT examinations were as follows: $80 \mathrm{kVp}, 100-$ $240 \mathrm{mAs}$, and $0.6 \mathrm{~mm}$ slice thickness. The gantry rotation time was 0.5 seconds, the collimation was $128 \times$ $0.6 \mathrm{~mm}$, and the matrix was $512 \times 512 \mathrm{~mm}$. Region of interest (ROI) was set in the ascending aorta keeping Hounsfield units (HU) of 80 . The 2D and 3D reconstructions in different planes were performed in maximum intensity projections. The measurements of the pulmonary valve, the main pulmonary artery, and branch pulmonary arteries were interpreted by a single radiologist with 14 years of experience after a review of each scan using a dedicated advanced workstation. The measurements of the pulmonary valve, the main pulmonary artery, and branch pulmonary arteries used in this study were derived after a review of each scan using the dedicated advanced workstation as per standard practice [9]. The radiologist was blinded with findings and measurements of TTE. On axial images at the level of bifurcation of a main pulmonary artery, the transverse diameter of the main pulmonary artery was taken in the image where the whole pulmonary trunk from annulus till bifurcation was visualized utilizing angulated multiplanar reformation (Figure 1) [10]. The branch pulmonary arteries were measured on axial images at a level of maximum diameter before branching (Figure 1). The pulmonary valvular level measurements were done from acquired CT image data by postprocessing and obtaining sagittal MIP reformation at a level where the right ventricle and main pulmonary artery were visualized [11]. 


\section{Cureus}

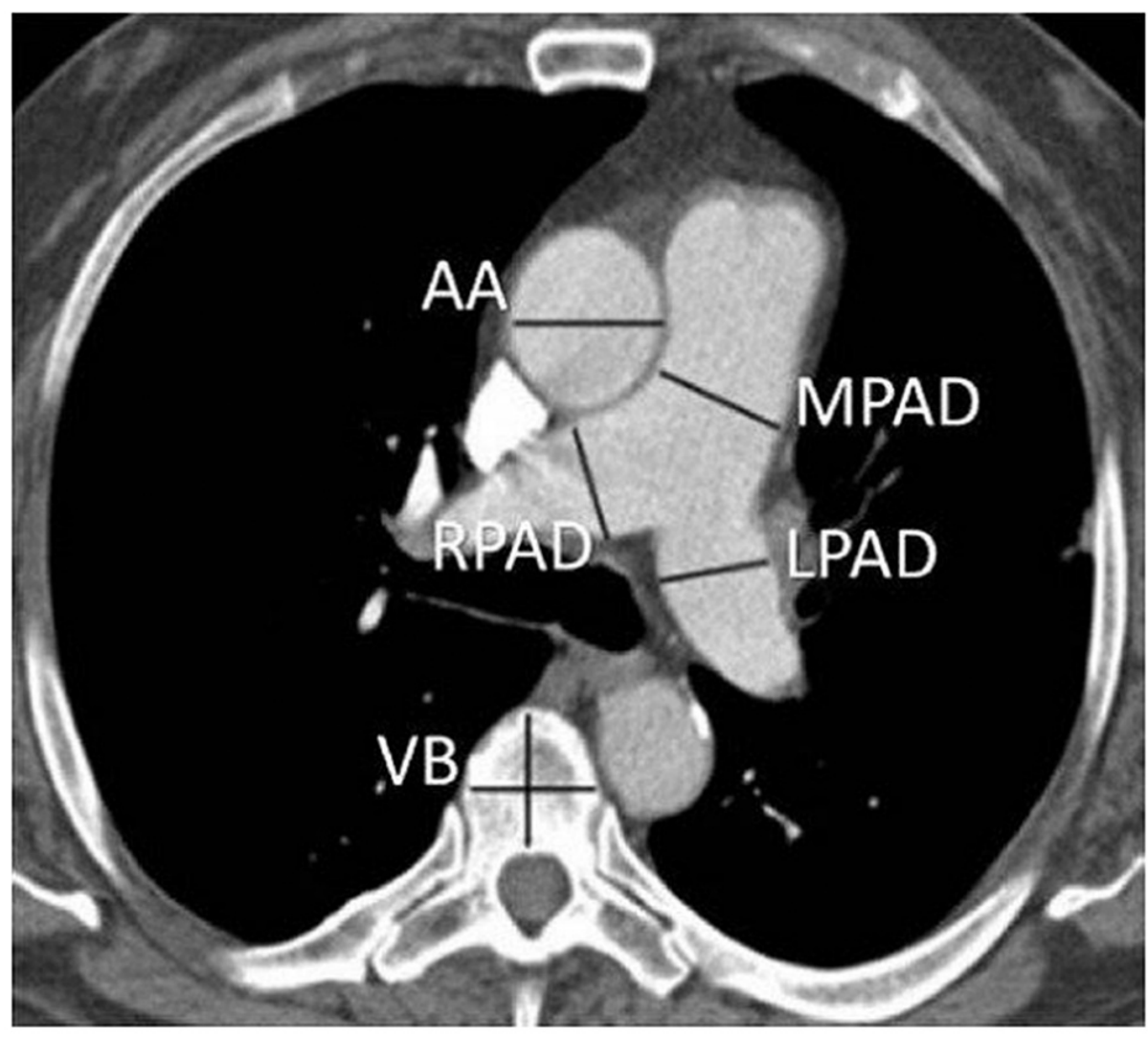

FIGURE 1: Axial image at the level of bifurcation of a main pulmonary artery

AA, Ascending aorta; MPAD, main pulmonary artery diameter; LPAD, left pulmonary artery diameter; RPAD, right pulmonary artery diameter; VB, vertebral body.

\section{Statistical analysis}

Descriptive statistics [mean (SD), frequency (\%)] were used to depict the profile of participants. The agreement between different parameters was assessed using Bland-Altman analysis. The normality of the differences was assessed by histogram. The agreement was presented as a mean difference ( $95 \%$ confidence limits). The analysis was performed using Stata 14.2 (StataCorp, College Station, TX, USA).

\section{Results}

A total of 49 patients (34 M, 15F) were enrolled in the study. Of these, $69 \%$ were males, and the mean [Median (Q1, Q3)] age of participants was 46.5 [18 $(5,81.3)]$ months. Almost half [22 $(46.9 \%)]$ of the participants were less than one-year old, while another quarter [13 (26.5\%)] of them were between one and five years of age. Cyanotic heart disease was present in 35 children who underwent MDCT scans in this study population. Tetralogy of Fallot, in its spectrum for atresia to absent pulmonary valve, was found in $48 \%$ of the patients (Table 1 ). 


\section{Cureus}

Diagnosis of congenital heart disease

Tetralogy of Fallot with pulmonary stenosis

Tetralogy of Fallot with pulmonary atresia

Tetralogy of Fallot with absent pulmonary valve

Total anomalous pulmonary venous connection

Scimitar syndrome

Atrial septal defect

Single ventricle with pulmonary stenosis

Pulmonary arteriovenous malformation

Pulmonary stenosis with intact ventricular septum

Single ventricle pulmonary hypertension

Ventricular septal defect with coarctation

Coarctation with patent ductus arteriosus

Double outlet right ventricle with pulmonary stenosis

Branch pulmonary artery stenosis

Single ventricle pulmonary atresia

Ventricular septal defect

Pulmonary embolism

Kawasaki syndrome

Total
$\%$

34.69

$4 \quad 8.16$

$3 \quad 6.12$

$3 \quad 6.12$

12.04

$3 \quad 6.12$

$4 \quad 8.16$

12.04

12.04

$2 \quad 4.08$

$1 \quad 2.04$

$2 \quad 4.08$

$2 \quad 4.08$

$1 \quad 2.04$

12.04

$1 \quad 2.04$

12.04

12.04

49

TABLE 1: Cardiac and pulmonary lesions in patients who underwent a CT scan and echocardiogram

CT, Computed tomography.

Bland-Altman analyses revealed the mean difference (95\% confidence limits) of diameter for the right pulmonary artery, measured on TTE and MDCT, was $-0.5(-3.1,2.2) \mathrm{mm}$ (Figure 2). Similarly, the mean difference (95\% confidence limits) for the diameter of the left pulmonary artery was $-0.6(-3.3,2.1) \mathrm{mm}$ (Figure 3). The mean difference (95\% confidence limits) diameter of the pulmonary annulus as measured on TTE and MDCT was $0.7(-2.5,3.9) \mathrm{mm}$ for the diameter of the pulmonary valve (Figure 4$)$. The difference was highest for the main pulmonary artery, $1.2(-6.9,4.5) \mathrm{mm}$ (Figure 5). The differences were without any upward/downward trend. 


\section{Cureus}
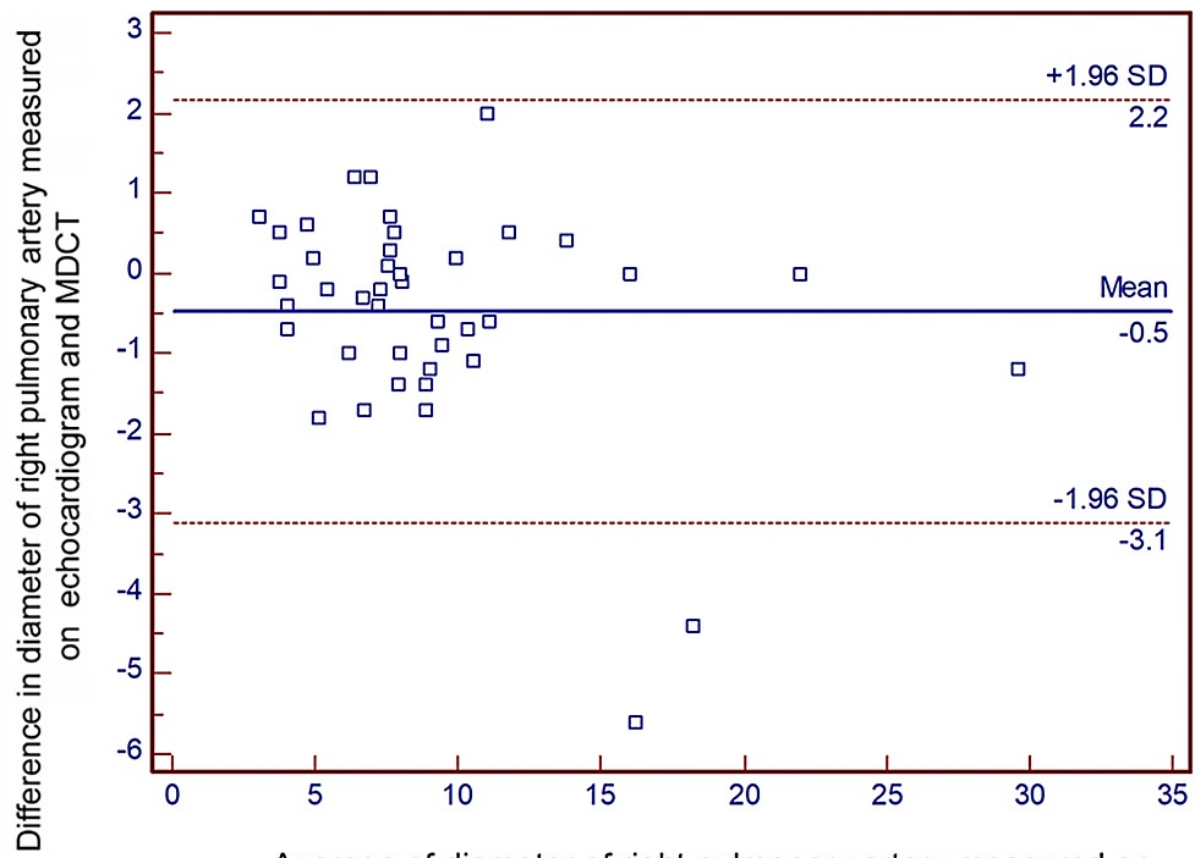

Average of diameter of right pulmonary artery measured on echocardiogram and CT Scan

FIGURE 2: Bland-Altman analysis for the diameter of the right pulmonary artery

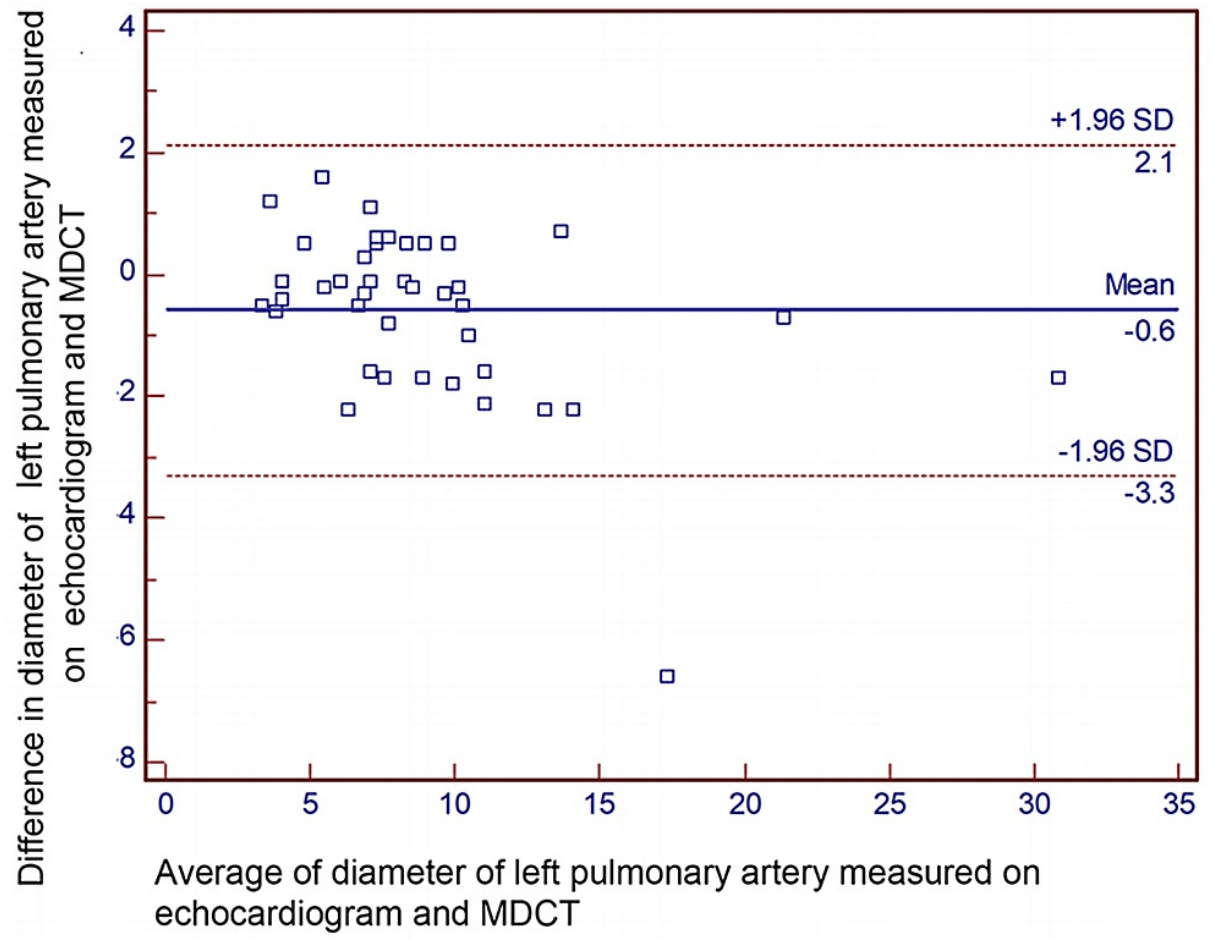

FIGURE 3: Bland-Altman analysis for the diameter of the left pulmonary artery 


\section{Cureus}

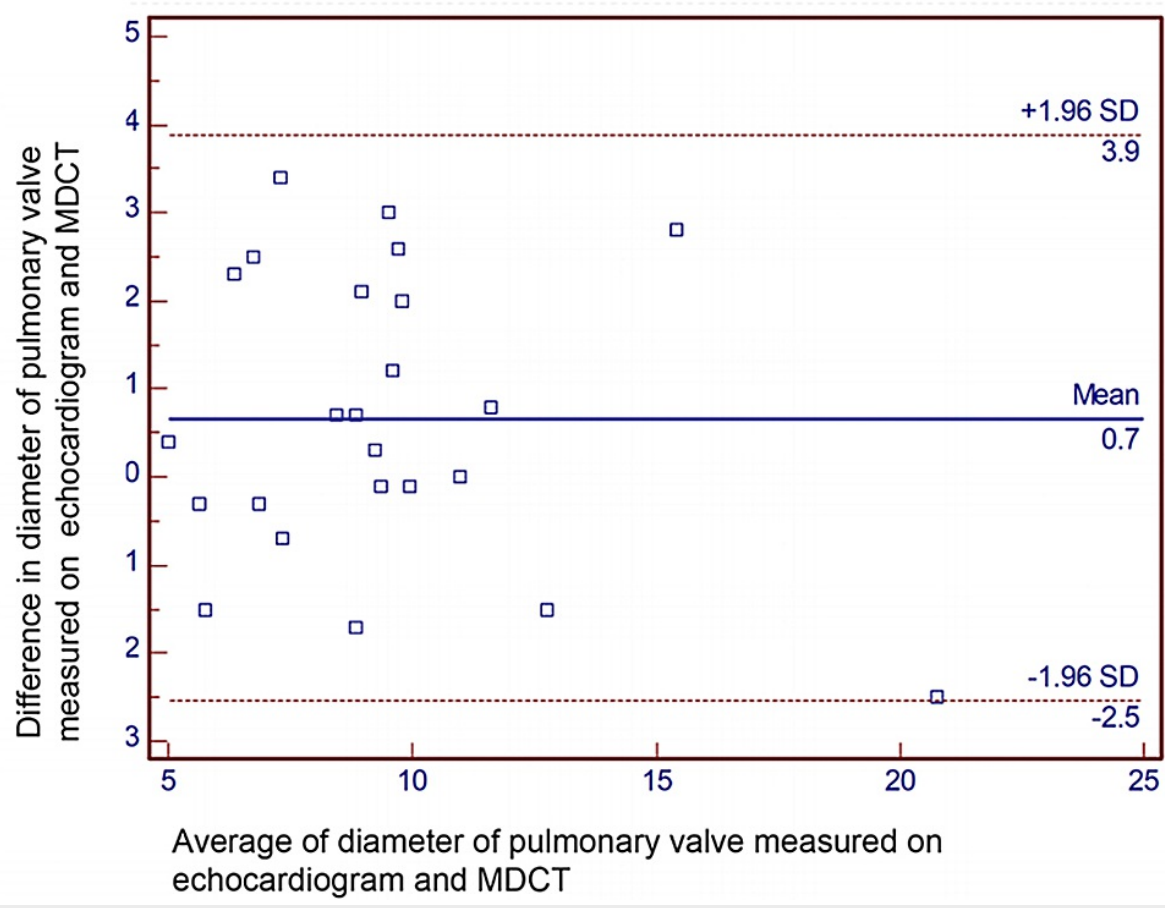

FIGURE 4: Bland-Altman analysis for the diameter of the pulmonary valve

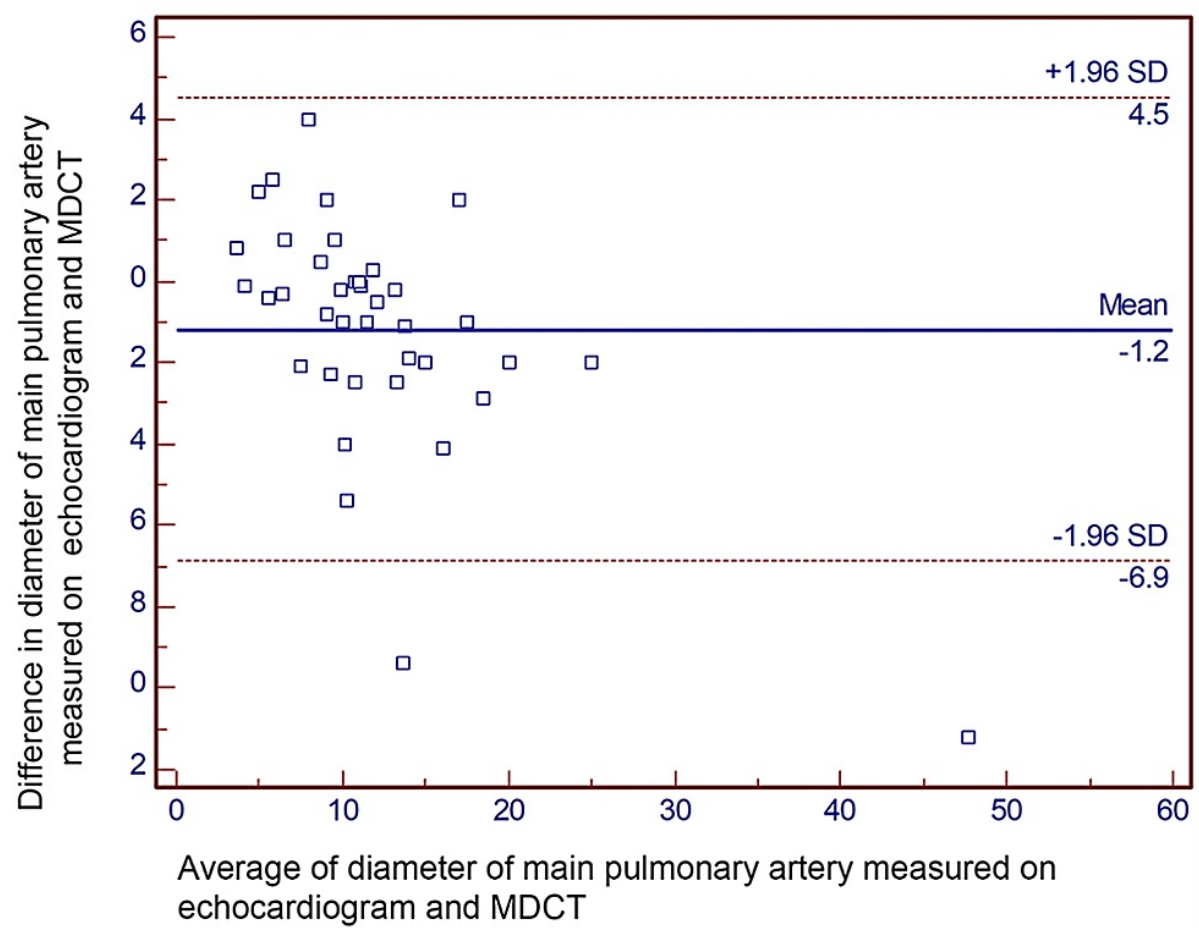

FIGURE 5: Bland-Altman analysis for the diameter of the main pulmonary artery

It was observed that out of 49 patients, 13 patients did not require surgical intervention. Out of 36 patients who were advised surgical intervention, 29 patients underwent surgeries. In 27 patients, the surgical plan remained the same after the MDCT scan as it was decided on an echocardiogram prior to the CT scan. In two patients, a surgical plan could not be decided on an echocardiogram alone; their plan was finalized after the CT scan. 


\section{Discussion}

Most children born with congenital heart disease require intervention, and the nature of the intervention is based on the anatomic details and hemodynamic conditions. TTE is used as a diagnostic tool for congenital heart disease since 1967 [12,13]. It is well established that TTE provides sufficient information for intracardiac structures, i.e., cardiac valves, chamber dimensions, intracardiac shunts, and hemodynamics $[14,15]$; however, due to its limitation with acoustic window, the extracardiac structures, i.e., distal pulmonary arteries, branch of the aorta, and individual pulmonary veins, are not very well visualized. Thus MDCT scan has been a preferred modality for pulmonary artery details when TTE is unequivocal [16].

It was noted in our study that for those who required surgical intervention - corrective or palliative - the provisional surgical plan based on echocardiogram did not change after the MDCT scan if the echocardiogram study provided sufficient information. There were two cases (a five-month-old male child with Scimitar syndrome and a 12-day-old male child with tetralogy of Fallot [TOF] with pulmonary atresia) where the TTE findings were unequivocal for surgical planning and MDCT provided details for surgical planning.

The study showed that the diameter of the pulmonary valve, main pulmonary artery, and branch pulmonary arteries measured on TTE and MDCT pulmonary angiogram were comparable.

The pulmonary valve annulus is one of the determinants to decide the type of corrective surgery for TOF, i.e., intracardiac repair with valve-sparing or trans-annular patch. The annular sparing surgeries carry preferable early surgical outcomes [6]. Our study shows the difference between the mean size of pulmonary valve annulus measured between echocardiogram and CT scan to be $0.7 \mathrm{~mm}$, similar to the study by Sathio et al. [17].

Complete correction for TOF cannot be done if the branch pulmonary arteries are smaller in size. Z score of pulmonary arteries is essential to decide the adequacy of branch pulmonary arteries for complete repair [18]. Earlier, cardiac catheterization and angiography were considered the gold standard for measuring right ventricular outflow tract components - starting from the subpulmonary region, pulmonary valve, main pulmonary artery, and branch pulmonary arteries [19]. Multidetector row computed tomography gained popularity as it is a significantly less invasive procedure while giving measurements comparable to an angiogram. A study of 56 MDCT scans for measurement of pulmonary arteries showed an excellent correlation between MDCT and conventional angiogram [20]. The current study results proved the comparability of measurements of the pulmonary arteries between TTE and MDCT. For measurement of pulmonary arteries using MDCT and TTE, the mean difference was being less than $1 \mathrm{~mm}$ for both branch pulmonary arteries, thus suggesting TTE be the first-line investigation and MDCT to be reserved for some instances due to its associated risk of radiation.

A 13-year review of surgical outcomes of 598 children of TOF suggested that TTE alone was sufficient to pick up anomalous coronary anatomy without the need for additional imaging [21]. The proportion of TOF patients undergoing cardiac surgery based on only TTE was $11 \%$, which went up to $82 \%$ between 1990 and 1995 due to advancements in technology [21]. MDCT scan remains the gold standard to study the complex coronary anatomy when an echocardiogram is not very informative. MDCT is also the modality of choice for complex anatomies such as aortopulmonary collaterals in the pulmonary atresia setting where native branch pulmonary arteries are either diminutive or may be completely absent. The treatment plan for such children needs MDCT [22].

The current study also showed good parity between the mean measurements of the pulmonary valve, measured on TTE and MDCT with a mean difference of $0.7 \mathrm{~mm}$. Most of our TTE was done under sedation allowing the pediatric cardiologist to perform a detailed echocardiogram.

The pulmonary valve is situated behind the sternum, sometimes making it difficult to assess on TTE. Due to its anterior position, trans-esophageal echocardiogram (TEE) also does not yield satisfactory images. In such cases, MDCT or MRI is necessary for evaluating the pulmonary valve [23]. TTE is the primary modality of choice to assess hemodynamics, and thus, it is used to assess the severity of pulmonary stenosis or regurgitation, while MDCT is necessary to evaluate the geometry and morphometry of RVOT [24].

TTE machines have undergone significant upgrades, starting from M Mode to two-dimensional imaging and three-dimensional imaging [25]. Newer echocardiogram machines are even equipped with true confocal image (TCI) and adaptive contrast enhancement (ACE) technologies. They help in acquiring high-resolution images and also help in differentiating between different tissues [26].

\section{Limitation}

The sample size and the retrospective nature of the study itself might have some inherent biases. The echocardiogram and MDCT both are operator-dependent. Similar results may not be reproducible at another center. CT scan also excels in studying the lobar branches and beyond the pulmonary tree. 


\section{Conclusions}

We conclude that the measurements of the pulmonary valve, main pulmonary artery, and branch pulmonary arteries in children obtained from the TTE study are comparable with MDCT. Hence, MDCT can be reserved for certain complex cases. It can help reduce exposure to radiation due to MDCT and reduce the treatment cost while ensuring the quality of care. We believe that more emphasis should be placed on performing a comprehensive echocardiogram by an experienced pediatric cardiologist.

\section{Additional Information \\ Disclosures}

Human subjects: Consent was obtained or waived by all participants in this study. Institutional Ethics Committee - 2, H.M. Patel Centre for Medical Care and Education, Karamsad, India issued approval IEC/HMPCMCE/2019/Ex. 14/. Your research proposal 'Comparison of right and left pulmonary artery dimensions measured in Helical CT Scan and Pediatric Echo-cardiogram in 75 children' was submitted for review and approval by committee members under Exempt Review. As it involves the collection of data from routine hospital practices/records with the maintenance of privacy and confidentiality, it qualified for an Exempt from Full Committee Review. The matter was reviewed by Committee Members and decided to review it under 'Exempt from full committee' review. After review and subsequent clarification by you, the project is approved by IEC in its present form with a waiver of consent for data collection. Animal subjects: All authors have confirmed that this study did not involve animal subjects or tissue. Conflicts of interest: In compliance with the ICMJE uniform disclosure form, all authors declare the following: Payment/services info: All authors have declared that no financial support was received from any organization for the submitted work. Financial relationships: All authors have declared that they have no financial relationships at present or within the previous three years with any organizations that might have an interest in the submitted work. Other relationships: All authors have declared that there are no other relationships or activities that could appear to have influenced the submitted work.

\section{References}

1. van der Linde D, Konings EE, Slager MA, Witsenburg M, Helbing WA, Takkenberg JJ, Roos-Hesselink JW: Birth prevalence of congenital heart disease worldwide: a systematic review and meta-analysis . J Am Coll Cardiol. 2011, 58:2241-7. 10.1016/j.jacc.2011.08.025

2. Bernier PL, Stefanescu A, Samoukovic G, Tchervenkov CI: The challenge of congenital heart disease worldwide: epidemiologic and demographic facts. Semin Thorac Cardiovasc Surg Pediatr Card Surg Annu. 2010, 13:26-34. 10.1053/j.pcsu.2010.02.005

3. Benavidez OJ, Gauvreau K, Jenkins KJ, Geva T: Diagnostic errors in pediatric echocardiography: development of taxonomy and identification of risk factors. Circulation. 2008, 117:2995-3001. 10.1161/CIRCULATIONAHA.107.758532

4. Ng AC, Delgado V, van der Kley F, et al.: Comparison of aortic root dimensions and geometries before and after transcatheter aortic valve implantation by 2- and 3-dimensional transesophageal echocardiography and multislice computed tomography. Circ Cardiovasc Imaging. 2010, 3:94-102. 10.1161/CIRCIMAGING.109.885152

5. Tops LF, Wood DA, Delgado V, et al.: Noninvasive evaluation of the aortic root with multislice computed tomography implications for transcatheter aortic valve replacement. JACC Cardiovasc Imaging. 2008, 1:32130. 10.1016/j.jcmg.2007.12.006

6. Azzam ME, Mohamed AS, Shawky HA, et al.: Early outcome of fallot repair with preservation of the pulmonary valve annulus versus transannular patch. SPER. 2018, 8:1-6.

7. Mercan AS, Sezgin A, Tokel K, et al.: The role of pulmonary artery anatomy in repair of tetralogy of fallot . Turk J Pediatr. 2001, 43:34-37.

8. Lopez L, Colan SD, Frommelt PC, et al.: Recommendations for quantification methods during the performance of a pediatric echocardiogram: a report from the Pediatric Measurements Writing Group of the American Society of Echocardiography Pediatric and Congenital Heart Disease Council. J Am Soc Echocardiogr. 2010, 23:465-95; quiz 576-7. 10.1016/j.echo.2010.03.019

9. Truong QA, Massaro JM, Rogers IS, et al.: Reference values for normal pulmonary artery dimensions by noncontrast cardiac computed tomography: the Framingham Heart Study. Circ Cardiovasc Imaging. 2012, 5:147-54. 10.1161/CIRCIMAGING.111.968610

10. Chen SJ, Huang JH, Lee WJ, Lin MT, Chen YS, Wang JK: Diagnosis of pulmonary arterial hypertension in children by using cardiac computed tomography. Korean J Radiol. 2019, 20:976-84. 10.3348/kjr.2018.0673

11. Chen JJ, Manning MA, Frazier AA, Jeudy J, White CS: CT angiography of the cardiac valves: normal, diseased, and postoperative appearances. Radiographics. 2009, 29:1393-412. 10.1148/rg.295095002

12. Ultan LB, Segal BL, Likoff W: Echocardiography in congenital heart disease: preliminary observations . Am J Cardiol. 1967, 19:74-83. 10.1016/0002-9149(67)90264-0

13. Moss AJ, Gussoni CC, Isabel-Jones J: Echocardiography in congenital heart disease. West J Med. 1976, 124:102-21.

14. Raimondi F, Warin-Fresse K: Computed tomography imaging in children with congenital heart disease: indications and radiation dose optimization. Arch Cardiovasc Dis. 2016, 109:150-7. 10.1016/j.acvd.2015.11.003

15. Li A, Peng Z, Zhang C: Comparison of echocardiography and 64-multislice spiral computed tomography for the diagnosis of pediatric congenital heart disease. Med Sci Monit. 2017, 23:2258-66. 10.12659/msm.901546

16. Shi K, Yang ZG, Xu HY, Zhao SX, Liu X, Guo YK: Dual-source computed tomography for evaluating pulmonary artery in pediatric patients with cyanotic congenital heart disease: comparison with 


\section{Cureus}

transthoracic echocardiography. Eur J Radiol. 2016, 85:187-92. 10.1016/j.ejrad.2015.11.002

17. Sathio SN, Shaikh AS, Korejo H, et al.: Comparison of size of pulmonary artery and its branches on transthoracic echocardiography versus computed tomographic angiography in patients with tetralogy of fallot. Cureus. 2020, $12:$ :9060. 10.7759/cureus.9060

18. Sinha R, Gooty V, Jang S, Dodge-Khatami A, Salazar J: Validity of pulmonary valve z-scores in predicting valve-sparing tetralogy repairs-systematic review $\uparrow$. Children (Basel). 2019, 6:67. 10.3390/children6050067

19. Feltes TF, Bacha E, Beekman RH 3rd, et al.: Indications for cardiac catheterization and intervention in pediatric cardiac disease: a scientific statement from the American Heart Association. Circulation. 2011, 123:2607-52. 10.1161/CIR.0b013e31821b1f10

20. Hayabuchi Y, Mori K, Kitagawa T, Inoue M, Kagami S: Accurate quantification of pulmonary artery diameter in patients with cyanotic congenital heart disease using multidetector-row computed tomography. Am Heart J. 2007, 154:783-8. 10.1016/j.ahj.2007.06.004

21. Need LR, Powell AJ, del Nido P, Geva T: Coronary echocardiography in tetralogy of fallot: diagnostic accuracy, resource utilization and surgical implications over 13 years. J Am Coll Cardiol. 2000, 36:1371-1377. 10.1016/S0735-1097(00)00862-7

22. Rajeshkannan R, Moorthy S, Sreekumar KP, Ramachandran PV, Kumar RK, Remadevi KS: Role of 64-MDCT in evaluation of pulmonary atresia with ventricular septal defect. AJR Am J Roentgenol. 2010, 194:110-8. 10.2214/AJR.09.2802

23. Han BK, Moga FX, Overman D, Carter C, Lesser JR: Diagnostic value of contrast-enhanced multiphase computed tomography for assessment of percutaneous pulmonary valve obstruction. Ann Thorac Surg. 2016, 101:e115-6. 10.1016/j.athoracsur.2015.09.096

24. Saremi F, Gera A, Ho SY, Hijazi ZM, Sánchez-Quintana D: CT and MR imaging of the pulmonary valve . Radiographics. 2014, 34:51-71. 10.1148/rg.341135026

25. Lang RM, Mor-Avi V, Sugeng L, Nieman PS, Sahn DJ: Three-dimensional echocardiography: the benefits of the additional dimension. J Am Coll Cardiol. 2006, 48:2053-69. 10.1016/j.jacc.2006.07.047

26. Wang CL, Hung KC: Recent advances in echocardiography. J Med Ultrasound. 2017, 25:65-7. 10.1016/j.jmu.2017.03.010 